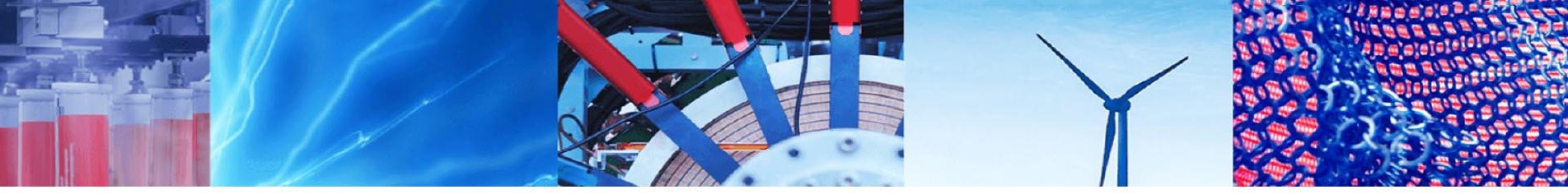

Research Article

\title{
Parameter estimation of laboratory-reared Panulirus ornatus
}

\author{
Chuan Hui Foo' ${ }^{1}$
}

Received: 6 February 2020 / Accepted: 26 March 2020 / Published online: 3 April 2020

(c) Springer Nature Switzerland AG 2020

\begin{abstract}
In the fisheries study, fish growth is typically in an indeterminate fashion implying a continuous growth function occurs throughout the life. Most existing studies of fish trajectories are continuous; however, it is not true when modelling growth of the crustacean species with discontinuous growth paths. In this context, it is imperative to insure that a different type of model for describing absolute growth in crustaceans. Crustaceans must moult for them to grow. A moulting process is of periodical shedding of the exoskeleton and thus the crustacean growth is known to be a discontinuous process. The sudden growth of crustaceans through the moulting process makes the growth estimation more complex. To model the discontinuous growth, we consider stochastic approaches where the growth model only considers for a monotonically increasing function. To this end, we introduce a subordinator that is a special case of a Levy process. A subordinator is a non-decreasing Levy process, that enabling the individual variability and environmental perturbation to be included in modelling growth. A dataset in the laboratory setting (e.g. in an aquarium) is developed. The motivational dataset is from the ornate rock lobster, Panulirus ornatus, where the growth parameters can be estimated through two inter-correlated variables, namely the intermoult periods and the moult increments. We propose a joint density function, consisting of the moult increments and the intermoult periods. Both of these variables are assumed to be conditionally independent based on the Markov property. In the four-year studies from 1995 to 1999, the growth rates for females and males are estimated averagely $0.307 \mathrm{~mm}_{\text {year }}{ }^{-1}$ and $0.205 \mathrm{~mm}_{\text {year }}{ }^{-1}$, respectively. Therefore, the growth parameters of moult increments and intermoult periods can be quantified individually. The corresponding functions will then be convoluted through a simulation approach to obtain a population mean curve for crustaceans.
\end{abstract}

Keywords Subordinator $\cdot$ Crustacean $\cdot$ Moult $\cdot$ Stochastic growth $\cdot$ Parameter estimation

\section{Introduction}

The most fundamental aspect of modeling growth rates of animals is to ensure optimal management for stock assessment. Modeling growth is important to determine the size-at-age or age-at-recruitment for sustainability in fisheries. Until recently, research on fish biology focused mainly on continuous growth study rather than on discontinuous growth processes [1].

It is important to take into consideration the needs of integrating discontinuity in growth because when crustaceans grow, they periodically shed their exoskeletons to make room for new exoskeletons $[9,11]$. However, some obstacles to moult-process estimation such as the stepwise function throughout the moulting cycle which caused difficulty in determining a crustacean growth at one time. Despite the challenges in modeling the discontinuous growth process, we can quantify the growth parameters via two important moult components: (1) moulting time interval (intermoult), and (2) moulting increment.

The purpose of this study is to describe mathematically the individual discontinuous trajectories of crustaceans by developing a new stochastic growth model that provide a

$\triangle$ Chuan Hui Foo, foo.ch@fsmt.upsi.edu.my | ${ }^{1}$ Department of Mathematics, Sultan Idris Education University, Tanjung Malim 35900 , Malaysia.

SN Applied Sciences (2020) 2:805 | https://doi.org/10.1007/s42452-020-2612-8 
few valuable insight by improving the existing stochastic growth models. To model individual moult increments, a Levy process can be considered in this context. A Levy process has right-continuous paths with left limits, indicating that there are countable finite 'jumps' in the growing process [7]. It generalises random walks to continuous time. However, there is a need to ensure a non-decreasing Levy process to characterize individual growth trajectories since the body size of an animal, practically, is unlikely to shrink throughout the moulting period. Many papers revised growth models based on stochastic differential equations, but some of the predictions may have non-positive increments [5] that may not be true. Consequently, one of the special cases of Levy process or more commonly known as a subordinator comes into play [12]. A subordinator is a Levy process with non-decreasing sample paths, it must have no diffusion component with almost surely non-decreasing paths and non-negative drift $[2,6]$. Specifically for subordinators which can only have non-negative jumps.

Talbot et al. [13] proposed a dynamic energy budget (DEB) model to describe the effect of environmental factors and food availability on the growth of crustacean species. Despite the environmental variability has been considered, the individual variability has yet to be thoroughly discussed in their model. In reality, each individual possesses its growth rate to reach the maximal length. The asymptotic carapace length in both sexes was inversely correlated to the growth rate, with a slower growth rate being recorded in the longer size of a crustacean [8]. For this reason, a biased estimation of growth will occur when there is high variability among individuals and has consistently been ignored [14]. Therefore, a subordinator can be very useful to model the randomness of the growth process due to individual and environmental variability.

\section{Materials and methods}

This study aims to account for stochasticity in modelling the discontinuous growth of crustaceans. Therefore, a subordinator is considered to describe non-decreasing measurement especially the moult increments in crustaceans. The most common examples from a class of subordinator are the gamma process and the Gaussian process [3].

Assuming that $Z_{t}$ is a subordinator. The Laplace transforms or the moment generating function of $Z_{t}[12]$ is expressed as

$$
\begin{aligned}
M_{t}(\theta) & =E\left(e^{-Z_{t}}\right) ; \quad \theta \geq 0 \\
& =e^{-t \varnothing(\theta)}
\end{aligned}
$$

where $e^{-t \varnothing(\theta)}$ is a Laplace exponent of the subordinator. Through this Laplace transform, the mean and variance of a subordinator can be estimated. For any Levy process, the first moment of the subordinator can be defined as

$E\left(Z_{t}\right)=\mu t$

whilst

$\operatorname{Var}\left(Z_{t}\right)=\sigma^{2} t$

in which $\mu$ is a finite mean and $\sigma^{2}$ is a finite variance for a Levy process.

The moult increments of crustacean species is modelled by using the gamma process, that is one of the classes of subordinators. The moment generating function of a gamma process is

$M_{t}(\theta)=\left(\frac{\lambda}{\lambda+\theta}\right)^{\alpha t}$.

Based on the above equation, the first and secondorder derivatives have been considered in quantifying the mean and variance of the gamma process such that

$E\left(Z_{t}\right)=\frac{\alpha t}{\lambda}$

and

$\operatorname{Var}\left(Z_{t}\right)=\frac{\alpha t}{\lambda^{2}}$.

As mentioned earlier, the discontinuous growth of crustaceans is determined by two fundamental stochastic components: (1) moult time interval (intermoult period), and (2) length increment between two consecutive moults (moult increment). Firstly, a joint density function will be developed using Markov property to estimate the growth parameters, knowing that both processes are conditionally independent.

Suppose an individual $i$ with $n_{i}$ repeated measures $\left\{L_{i, j-1}, T_{i, j}, I_{i, j}\right\}$ from $j=1$ to $n_{i}$ where $L_{i, j-1}$ is the $j$ th length before moulting, $T_{i, j}$ is the $j$ th intermoult period, and $I_{i, j}$ is the $j$ th moult increment. Assuming an animal is observed at different times, a general growth function is imperative to characterize the distribution of the sample paths for all animals. Let $f\left(L_{i, 0}\right)$ be an initial length function for an animal. Denote that $g(\cdot)$ as a function of intermoult periods and $h(\cdot)$ as a function of moult increments, given that both functions are conditioned on the premoult length and intermoult periods. Considering an animal $i$ moulted three times, a unified probability density function of intermoult periods and moult increments can be expressed as: 


$$
\begin{aligned}
& f\left[\left(L_{i 2}, T_{i 3}, I_{i 3}\right),\left(L_{i 1}, T_{i 2}, I_{i 2}\right),\left(L_{i 0}, T_{i 1}, I_{i 1}\right)\right] \\
& =g\left(T_{i 3} \mid L_{i 2}, T_{i 2}\right) g\left(T_{i 2} \mid L_{i 1}, T_{i 1}\right) g\left(T_{i 1} \mid L_{i 0}, T_{i 0}\right) \\
& \quad h\left(I_{i 3} \mid L_{i 2}, T_{i 3}\right) h\left(I_{i 2} \mid L_{i 1}, T_{i 2}\right) h\left(I_{i 1} \mid L_{i 0}, T_{i 1}\right) f\left(L_{i 0}\right) .
\end{aligned}
$$

In general, the joint density function for the $N$ individuals can be written as

$L(\theta)=\prod_{i=1}^{N}\left(\prod_{j=1}^{n_{i}}\left[g\left(T_{i j} \mid L_{i j-1}, T_{i j-1}\right)\right] \prod_{j=1}^{n_{i}}\left[h\left(I_{i j} \mid L_{i j-1}, T_{i j}\right) f\left(L_{i 0}\right)\right]\right)$

where the function $g$ refers to the intermoult periods function, whereas the function $h$ concerning the moult increments. Since both functions are assumed to be conditionally independent, therefore the likelihood functions can be estimated respectively. Eventually, both functions can be convoluted through Monte Carlo simulation to describe individual growth trajectories with a population mean for the crustaceans.

For modeling the moult increment $l_{t}$, let $l_{t}$ follows a gamma subordinator. If $Z_{t}, t \geq 0$ is a subordinator, then $I_{t}=Z_{t}$ with the mean of $I_{t}$ is assumed to follow a Faben's method [4] that is generalized from a von Bertalanffy growth function [15]. Therefore, the estimated moult increment is simplified as

$E\left(I_{t}\right)=\left(L_{\infty}-L^{-}\right)\left(1-e^{-k t}\right)$

where $t$ is the time interval between two consecutive moults, $L^{-}$is the premoult length, $k$ is the growth rate and $L_{\infty}$ is the estimated asymptotic length of an individual. Let the mean of moult increments follows a gamma distribution that $I_{t} \sim \Gamma(\alpha, \lambda)$, hence the expected of moult increments is

$E\left(I_{t}\right)=\frac{\alpha\left(L_{\infty}-L^{-}\right)}{\lambda}\left(1-e^{-k t}\right)$

and the variance of moult increments is

$\operatorname{Var}\left(I_{t}\right)=\frac{\alpha\left(L_{\infty}-L^{-}\right)}{\lambda^{2}}\left(1-e^{-k t}\right)$

From Eqs. (5) and (6), we notice that $\mu$ plays the role of $\alpha\left(L_{\infty}-L^{-}\right) / \lambda$ while $\sigma^{2}$ equals to $\alpha\left(L_{\infty}-L^{-}\right) / \lambda^{2}$.

For modelling the intermoult periods $T_{L}$, the parameter estimation is considerably simple since the moulting times can be detected as soon as an individual moulted from the tank. From the growth model in Restrepo [10], the estimated intermoult periods is a function of premoult length $L^{-}$. Restrepo presumed the intermoult periods follow a lognormal distribution that $\log \left(T_{L}\right) \sim N\left(\mu, \sigma^{2}\right)$, the mean of the intermoult period is hence

$$
\begin{aligned}
& E\left(T_{L}\right)=e^{\mu+\sigma^{2} / 2}=\alpha e^{\beta L^{-}}, \\
& \mu=\log (\alpha)-\sigma^{2} / 2+\beta L^{-} \\
& \text {where } \alpha>0 \text { and } \beta \geq 0 .
\end{aligned}
$$

\section{Results and discussion}

The dataset was collected in Cairns, Australia, from the year 1995 to 1999. A total of 75 lobsters, made up of 39 females and 36 males, were reared in tanks over a timespan of 4 years. Lobsters' growth were observed through their moult intervals and moult increments in general. The carapace lengths of the moulted exoskeleton were measured between $6.3 \mathrm{~mm}$ and $158.3 \mathrm{~mm}$ on the lobsters. The lengths increased continually for female and male lobsters in jumpy manner throughout the 4-year study as shown in Fig. 1.

Most of the lobsters moulted more frequently during their juvenile stage compared to adults. The bigger the size of a lobster, the longer time for a lobster to get moulted Fig. 2. Besides that, the moult increments increased along with the increasing function of intermoult periods Fig. 3.

The parameter estimates are sex-specified, showing a different set of parameters for males and females will be obtained. For modelling intermoult periods, the parameter estimates through optimization were obtained as in Table 1. The deviation of growth rates $\sigma$ is higher for female lobsters due to the stage of maturity and energy allocation purposes. Therefore, females grew slower to reach the asymptotic length compared to males. For modelling moult increments, when growth trajectories follow a gamma density function, both sets of $\left(\lambda, k, L_{\infty}\right)$ yield $(0.102,0.307,175.33)$ for females and $(0.617,0.205,258.72)$ for males, respectively. Male lobsters have greater asymptotic length $L_{\infty}$ than females Table 2.

Consequently, we convolute both moult increments and intermoult periods functions through Monte Carlo simulation. All the simulation studies are implemented in the $\mathrm{R}$ environment. We simulated 1000 iterations for a subordinator-based model with each sample size of 100 males and females. By incorporating both components of moult increments and intermoult periods, the stochastic growth trajectories for individuals can be characterized mathematically. Overall, both males and females displayed a monotonically increasing pattern and converged to a common $L_{\infty}$ when the time approached infinity Fig. 4 . Females possess a higher growth rate and a larger asymptotic length compared to males for the 4-year study. 
Fig. 1 Panulirus ornatus growth paths over the years

Fig. 2 Intermoult periods versus carapace length
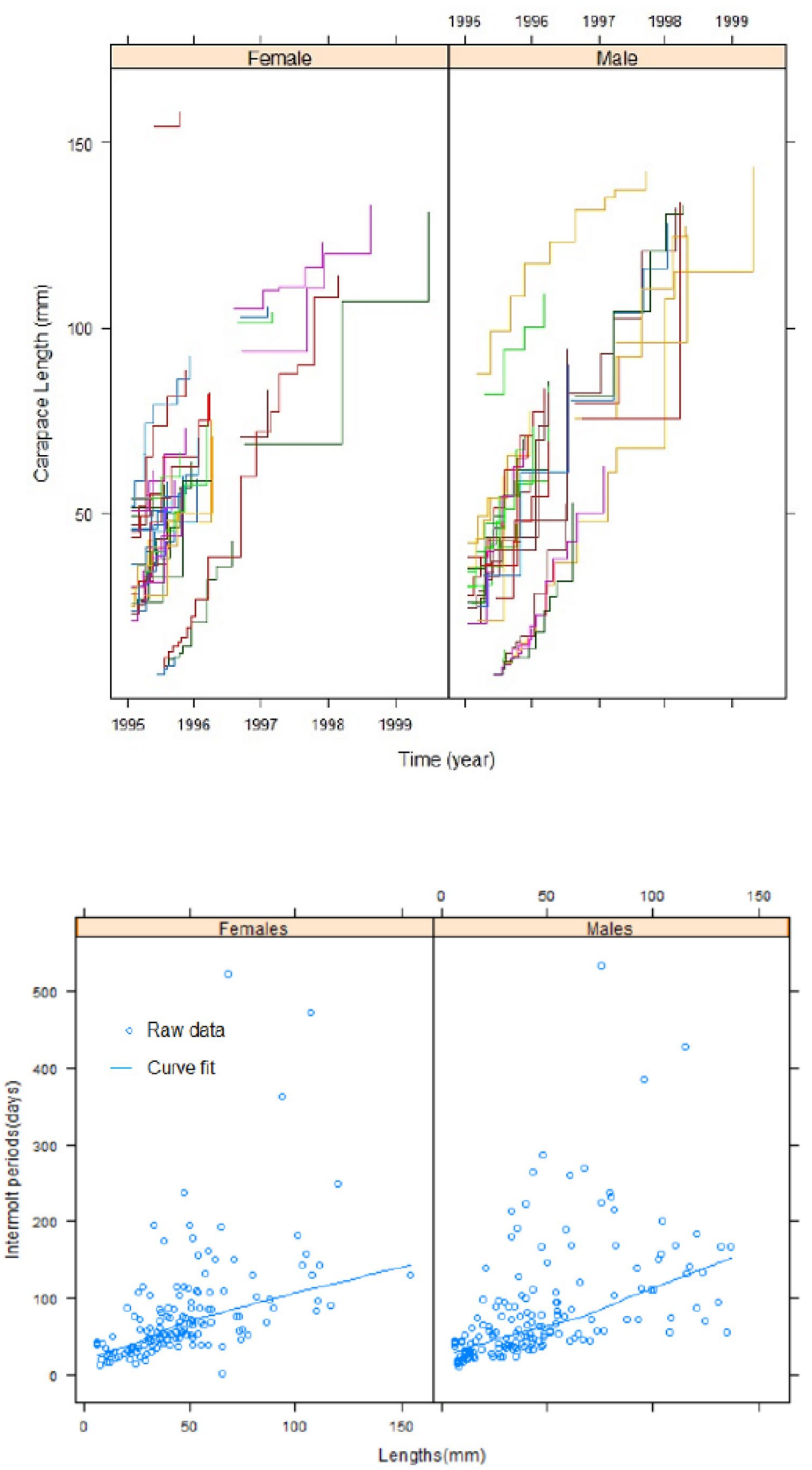
Fig. 3 Moult increments over time

Table 1 Parameter estimates for intermoult periods following a lognormal function

\begin{tabular}{llll}
\hline & $\alpha$ & $\beta$ & $\sigma$ \\
\hline Female & 0.102 & 0.015 & 0.637 \\
Male & 0.107 & 0.014 & 0.585 \\
\hline
\end{tabular}

Table 2 Parameter estimates for moult increments following a gamma distribution

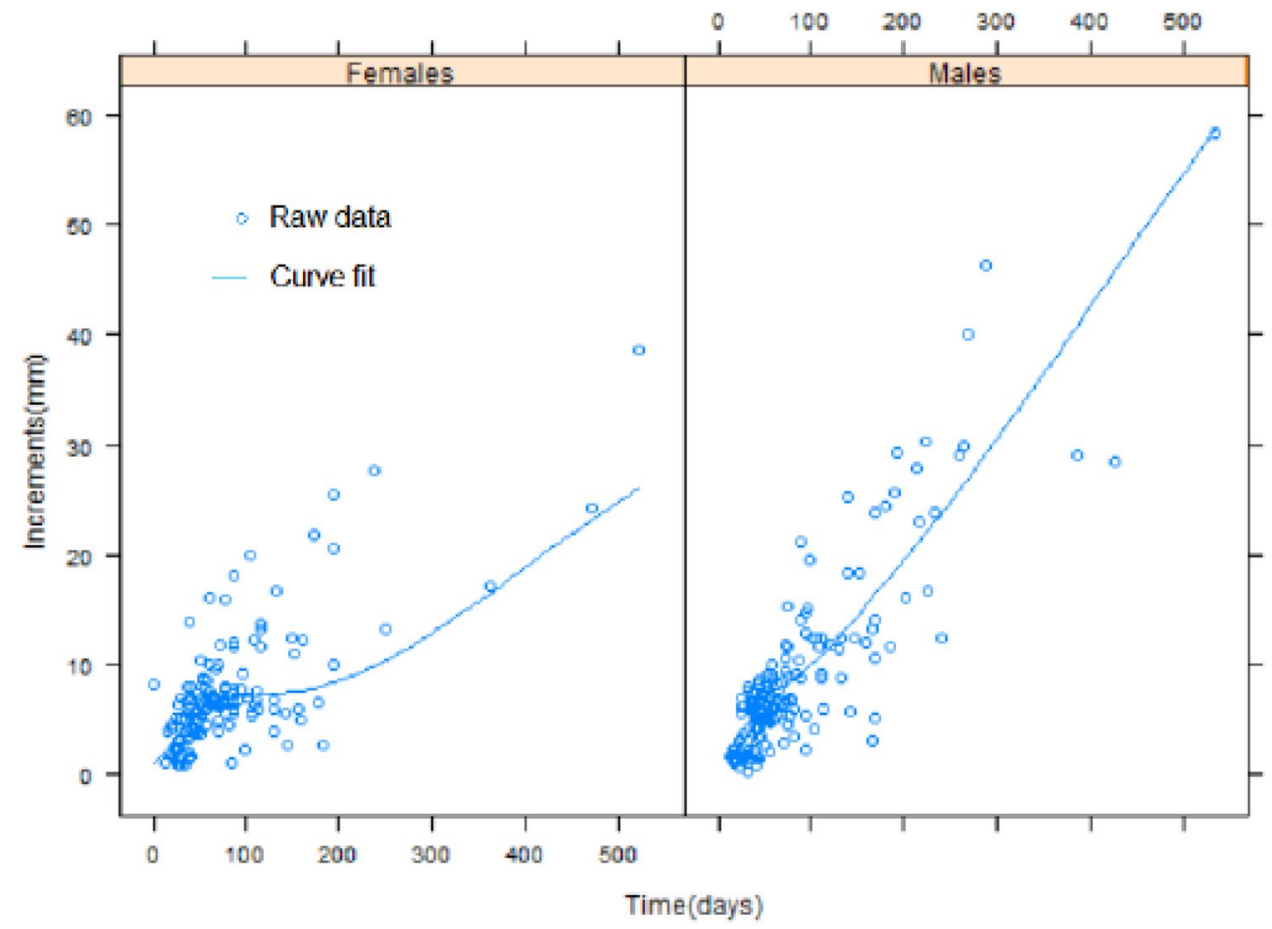

\section{Conclusions}

In the fishery industry, the populations of lobsters are prone to decline due to increasing demand as a delicacy worldwide. For the sake of sustainability, modeling growth is crucial in monitoring the stock assessment and to get rid of overfishing in fish farming.

Two stochastic components determine a moulting process: moult increments and intermoult periods. Since both components generate discontinuous growth paths, we consider stochastic growth model by adding subordinator

\begin{tabular}{llll}
\hline & $\lambda$ & $k$ & $L_{\infty}$ \\
\hline Female & 0.621 & 0.307 & 175.33 \\
Male & 0.617 & 0.205 & 258.72 \\
\hline
\end{tabular}

Gamma process for females

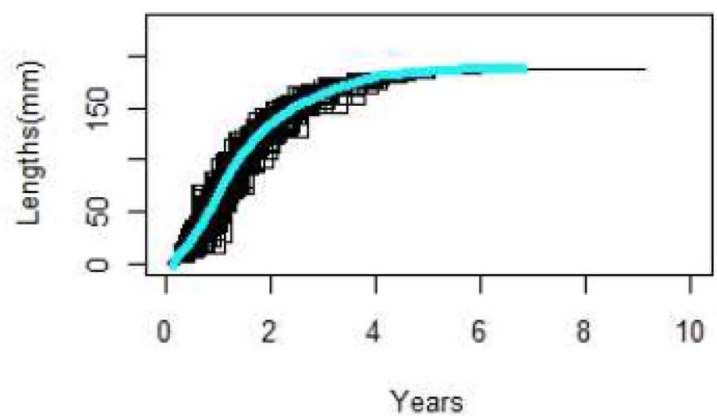

Gamma process for males

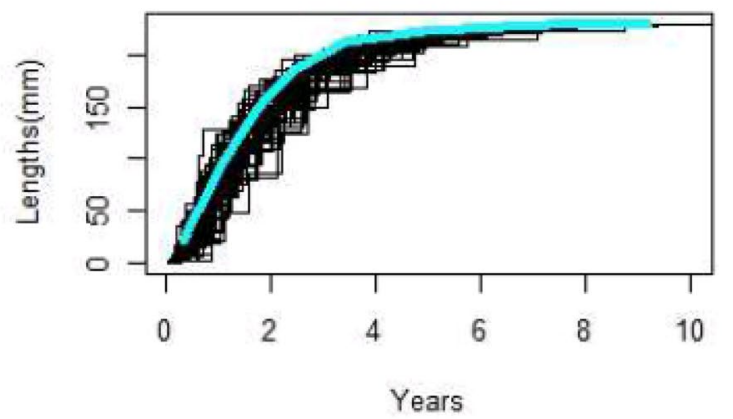

Fig. 4 Growth trajectories with population mean $L_{\infty}$ (blue line) 
into the proposed model as it incorporates individual as well as environmental variability for Panulirus ornatus population from laboratory experiments. Through this, we can characterize individual stepwise growth trajectories and thus produce a population mean curve for crustacean data.

\section{Compliance with ethical standards}

Conflict of interest The author declare that they have no competing interests

\section{References}

1. Chang YJ, Sun $C L$, Chen $Y$, Yeh SZ (2012) Modelling the growth of crustacean species. Rev Fish Biol Fish 22:157-187. https://doi. org/10.1007/s11160-011-9228-4

2. Cont R, Tankov P (2004) Financial modelling with jump processes. Chapman and Hall/CRC financial mathematics series. Taylor \& Francis, Routledge

3. Dassios A, Lim JW, Qu Y (2019) Exact simulation of truncated Levy subordinator. ACM Trans Model Comput Simul 56:57-75

4. Fabens AJ (1965) Properties and fitting of the von Bertalanffy growth curve. Growth 29:265-289

5. Garcia O (1983) A stochastic differential equation model for the height growth of forest stands. Biometrics. https://doi. org/10.2307/2531339

6. Jacob F (2015) Risk estimation on high frequency financial data: empirical analysis of the DAX 30 . Springer, Berlin

7. Jarrow RA (2018) Stochastic processes. In: Jarrow RA (ed) Continuous-time asset pricing theory. Springer Finance. Springer, Cham
8. Mendes-Junior RNG, Sá-Oliveira JC, Ferrari SF (2016) Biology of the electric eel, Electrophorus electricus, Linnaeus, 1766 (Gymnotiformes:Gymnotidae) on the floodplain of the Curiaú River, eastern Amazonia. Rev Fish Biol Fish 26:83-91. https://doi. org/10.1007/s11160-015-9407-9

9. Nguyen TV, Jung $H$, Rotlant $G$, Hurwood D, Mather P, Ventura T (2018) Guidelines for RNA-seq projects: applications and opportunities in non-model decapod crustacean species. Hydrobiologia. https://doi.org/10.1007/s10750-018-3682-0

10. Restrepo VR (1989) Growth estimates for male stone crabs along the Southwest Coast of Florida: a synthesis of available data and methods. Trans Am Fish Soc 118:20-29. https://doi. org/10.1577/1548-8659

11. Rotlland G, Palero F, Mather PB, Bracken-Grissom HD, Santos MB (2018) Preface: recent advances in crustacean genomics. Hydrobiologia 825:1-4. https://doi.org/10.1007/s10750-018-3773-y

12. Russo T, Baldi P, Parisi A, Magnifico G, Mariani S, Cataudella $S$ (2009) Levy processes and stochastic von Bertalanffy models of growth, with application to fish population analysis. J Theor Biol 258:521-529. https://doi.org/10.1016/j.jtbi.2009.01.033

13. Talbot SE, Widdicombe S, Hauton C, Bruggeman J (2018) Adapting the dynamic energy budget (DEB) approach to include noncontinuous growth (moulting) and provide better predictions of biological performance in crustaceans. ICES J Mar Sci 76:192205. https://doi.org/10.1093/icesjms/fsy164

14. Vincenzi S, Mangel M, Crivelli AJ, Munch S, Skaug HJ (2014) Determining individual variation in growth and its implication for life-history and population processes using the empirical Bayes method. PLoS Comput Biol 10:e1003828. https://doi. org/10.1371/journal.pcbi.1003828

15. Von Bertalanffy L (1938) A quantitative theory of organic growth. Hum Biol 10:181-213

Publisher's Note Springer Nature remains neutral with regard to jurisdictional claims in published maps and institutional affiliations. 